\title{
Establishment of a Novel Endothelial Target Mouse Model of a Typhus Group Rickettsiosis: Evidence for Critical Roles for Gamma Interferon and CD8 T Lymphocytes
}

\author{
David H. Walker, Vsevolod L. Popov, and Hui-Min Feng \\ Department of Pathology, Rickettsial and Ehrlichial Diseases Research Laboratory, University of Texas Medical \\ Branch, Galveston, Texas
}

\begin{abstract}
SUMMARY: A mouse model of typhus rickettsiosis that reproduces the hematogenous dissemination to the critical target organs, including brain, lungs, heart, and kidneys, primary endothelial and, to a lesser degree, macrophage intracellular rickettsial infection, and typical vascular-based lesions of louse-borne typhus and murine typhus was established. Intravenous inoculation of $\mathrm{C} 3 \mathrm{H} / \mathrm{HeN}$ mice with Rickettsia typhi caused disease with a duration of the incubation period and mortality rate that were dependent on the infective dose of rickettsiae. Lethal infection was associated with high concentrations of $R$. typhi in the lungs and brain, despite a brisker humoral immune response to the rickettsiae than in the sublethal infection. Gamma interferon and CD8 T lymphocytes were demonstrated to be crucial to clearance of the rickettsiae and recovery from infection in experiments in which specific monoclonal antibodies were administered to deplete these components. Death of animals depleted of gamma interferon or CD8 T lymphocytes was associated with overwhelming rickettsial infection demonstrated by titers of infectious rickettsiae and by immunohistochemistry. An effective antirickettsial immune response was associated with elevated serum concentrations of IL-12 on Day 5 and increased secretion of IL-12 by concanavalin-A-stimulated spleen cells on Day 5 . Evidence for transient suppression of the immune response consisted of marked reduction in the secretion of IL-2 and IL-12 by concanavalin-A-stimulated spleen cells on Days 10 and 15. This model offers excellent opportunities for study of attenuation and pathogenetic mechanisms of typhus rickettsiae, which are established biologic weapons of potential use in bioterrorism. (Lab Invest 2000, 80:1361-1372)
\end{abstract}

\begin{abstract}
Rickettsia typhi is the etiologic agent of murine Rtyphus, one of the most prevalent and least diagnosed infections of tropical and subtropical regions, typically coastal locations with a warm climate. Rickettsia prowazekii causes epidemic louse-borne typhus fever, one of the most important infectious diseases of the last 500 years from the perspective of its historic impact (Zinsser, 1935; Traub et al, 1978). These obligately intracellular bacteria are closely related genetically and share many antigenic and biologic characteristics (Vishwanath, 1991; Roux and Raoult, 1995; Roux et al, 1997; Stothard and Fuerst, 1995). Their shared antigens include the group-specific lipopolysaccharide and many epitopes of the major immunodominant surface protein. Unlike spotted fever group (SFG) rickettsiae, which stimulate the polymerization of F-actin tails that propel them through the host cell cytoplasm and enable them to spread from cell-tocell, these typhus group rickettsiae remain in the cytoplasm of the host cell even though a small fraction of $R$. typhi do stimulate host cell generation of short
\end{abstract}

Received April 10, 2000.

Address reprint requests to: Dr. David H. Walker, Professor and Chairman, Department of Pathology, 301 University Boulevard, Galveston, TX 77555-0609. Fax: 409772 2500; E-mail: dwalker@utmb.edu
F-actin tails (Wisseman and Waddell, 1975; Wisseman et al, 1976; Teysseire et al, 1992; Heinzen et al, 1993). They divide repeatedly by binary fission until the distended cell bursts. Previously, two animal models of $R$. typhi infection have been described: guinea pigs inoculated intraperitoneally, intradermally, or intravenously and Balb/c mice inoculated subcutaneously or intravenously (Murphy et al, 1978, 1979; Crist et al, 1984a, 1984b). Intradermally inoculated guinea pigs develop an eschar at the site of inoculation and local growth of rickettsiae followed by spread to and growth in the regional lymph node and hematogenous dissemination to the spleen and kidney (Murphy et al, 1978). Although intraperitoneally inoculated guinea pigs develop febrile rickettsial peritonitis, animals inoculated intradermally or intravenously develop neither fever nor other signs of illness, and there is no fatal course (Murphy et al, 1978, 1979). Although the intradermal model was useful in demonstrating the importance of cellular immunity by adoptive transfer of immune spleen cells, the model was not characterized by identification of the target cells or any histologic lesions (Murphy et al, 1979). Similarly in Balb/c mice inoculated subcutaneously or intravenously, no illness or fatalities occurred, and the cells infected with rickettsiae and presence of histopathologic lesions were not described. Research on the pathogenesis of 
typhus and the mechanisms of immunity to typhus group rickettsiae has been constrained by the lack of a reproducible animal model in which the endothelial cell is the major target and the vascular pathology resembles the human typhus fevers. There has been no consensus that immune mechanisms elucidated for SFG rickettsioses apply to typhus group rickettsial infections (Feng et al, 1994, 1997). Thus, this investigation was undertaken to develop an endothelial target model of typhus rickettsiosis in a genetically defined strain of mice that develop clinical illness, characteristic rickettsial histopathology, and dosedependent fatal or non-fatal outcome, and to assess the role of gamma interferon and CD8 T lymphocytes in rickettsial clearance and recovery from infection with $R$. typhi.

\section{Results}

Morbidity, Mortality, and Rickettsial Content of Organs in Mice Receiving a Lethal or Sublethal Dose of R. typhi

All of the mice inoculated with 10 median lethal doses $\left(\mathrm{LD}_{50}\right)$ of $R$. typhi became ill after 3 days. One died on Day 5 , three died on Day 6, and six moribund mice were killed on Day 6. There were no survivors. All of the mice inoculated with $0.1 L_{50}$ became ill after 7 days, none died, and all six mice that had not been killed on schedule recovered on Day 13. Infectious rickettsiae were first recovered from the lethally infected mice on Day 2, and increased rickettsial titers were observed in all organs tested on Day 6 (Table 1). The rickettsial titers on Days 5 and 10 in the sublethally infected mice were lower than those in the lethally infected mice on Days 4 and 6. By Day 15, when the sublethally inoculated mice seemed to have recovered completely, the content of $R$. typhi in the spleen was less than $5 \%$ of the quantity of infectious rickettsiae that had been present 10 days earlier. Despite a brisker and higher rise in titer of antibodies to $R$. typhi, the mice inoculated with the higher rickettsial dose died nonetheless (Table 1).

\section{Immunohistochemical and Ultrastructural Evaluation of the Distribution of Typhus Group Rickettsiae in Mice Receiving a Lethal Dose of R. typhi}

Typhus group rickettsiae were detected in endothelial cells of pulmonary alveolar capillaries and renal intertubular capillaries and in macrophages in the marginal zone and red pulp of the spleen on Day 2 after inoculation. The liver also contained $R$. typhi organisms within macrophages and polymorphonuclear leukocytes of multifocal granuloma-like lesions, as well as in numerous sinusoidal lining cells without an associated inflammatory reaction. Focal rickettsiae were also detected in capillary endothelium of the myocardium. On Days 4 and 6, focal typhus group rickettsial infections were also identified in cerebral endothelial cells (Fig. 1A).

On Days 4 and 6 , a progressive increase in the quantity of rickettsiae was noted in pulmonary alveolar capillaries (Fig. 1B), cerebral and meningeal capillaries, renal intertubular capillaries, myocardial capillaries, and hepatic sinusoidal lining cells.

The severe vascular infection on Days 4 and 6 was not associated with a cellular immune or inflammatory response. Focal areas of cerebral endothelial cells were strikingly swollen, corresponding to massive distension by intracellular rickettsiae (Fig. 2). On Day 6 , multifocal endothelial infection of capillaries was observed in the lamina propria of the small intestine and in the interstitium of the testis.

Electron microscopy identified rickettsiae in the cytoplasm of endothelial cells in the lung and in macrophages in the spleen and in Kupffer cells in the liver on Day 2 after inoculation (Fig. 3). On Day 4, the quantity of rickettsiae had increased, resulting in cells distended with massive quantities of cytoplasmic organisms (Figs. 4 and 5). The rickettsial organisms showed two different morphologic appearances, light and dense protoplasm. Many rickettsiae contained vacuoles within their cytoplasm, a typical feature of typhus group rickettsiae (Fig. 6). Some nonviableappearing rickettsiae were observed in phagolysosomes of macrophages (Fig. 7).

\section{Course, Histopathology, and Immune Response of C3H/HeN Mice Inoculated Intravenously with 0.88 $L D_{50}$ of $R$. typhi}

All 30 sham-depleted mice that were inoculated with R. typhi became ill on Day 8 or 9 with decreased activity, ruffled fur, hunched posture, and partially closed eyes. Among the 14 sham-depleted mice that were observed for 15 days, $43 \%$ succumbed to the infection with death on Day 10 (two mice), 12 (one mouse), or 13 (three mice) (Table 2). On Day 10, $R$. typhi infection remained present in the lungs, spleen,

Table 1. Infectious Rickettsial Content ${ }^{a}$ and Antibody Titer in Mice Infected with a Lethal or Sublethal Dose of $R$. Typhi

\begin{tabular}{cccccc}
\hline $\begin{array}{c}\text { Dose of } \\
\text { inoculum }\end{array}$ & $\begin{array}{c}\text { Day after } \\
\text { inoculation }\end{array}$ & Spleen & Lungs & Brain & IFA titer \\
\hline $10 \mathrm{LD}_{50}$ & 2 & $0.5 \pm 0.5$ & $0.32 \pm 0.32$ & $0.14 \pm 0.14$ & $<1: 40$ \\
& 4 & $182.5 \pm 3.6$ & $8.6 \pm 7.7$ & $5.7 \pm 5.8$ & $1: 640$ \\
& 6 & $226.7 \pm 23.6$ & $41.7 \pm 26.5$ & $24.0 \pm 6.5$ & $1: 640$ \\
$0.1 \mathrm{LD}_{50}$ & 5 & $17.8 \pm 0.2$ & $3.3 \pm 1.7$ & $0.5 \pm 0.3$ & $1: 40$ \\
& 10 & $10.02 \pm 9.4$ & $2.6 \pm 0.6$ & $0.45 \pm 0.45$ & $1: 320$ \\
& 15 & $0.8 \pm 0.9$ & $2.1 \pm 2.1$ & $0.24 \pm 0.24$ & $1: 320$ \\
\hline
\end{tabular}

${ }^{a} \operatorname{TCID}_{50} \times 10^{4}$ per gram of tissue; means of 2 to 3 repeated experiments. 


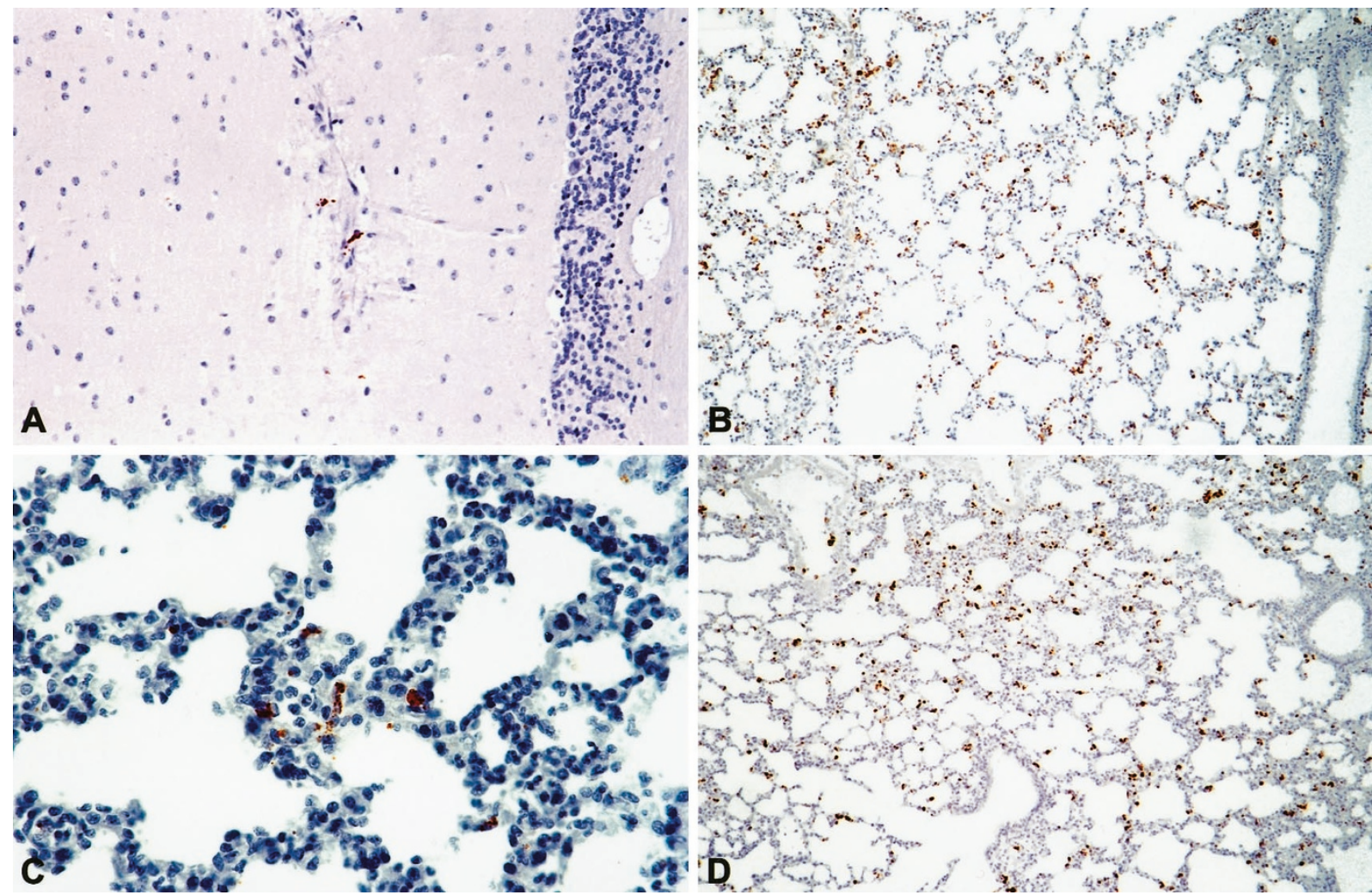

\section{Figure 1.}

Monoclonal antibody-based immunoperoxidase demonstration of Rickettsia typhi in tissues of experimentally infected $\mathrm{C} 3 \mathrm{H} / \mathrm{HeN}$ mice. A, Brain of a mouse infected with $10 \mathrm{LD}_{50}$ of $R$. typhi 4 days earlier shows vascular endothelial cells distended with cytoplasmic rickettsiae; original magnification, $\times 105$. B, Lung of a moribund mouse inoculated 6 days earlier with $10 \mathrm{LD}_{50}$ of $R$. typhi shows massive quantities of rickettsiae distributed throughout the microcirculation; original magnification, $\times 53$. C, Lung of a sham-depleted mouse 10 days after inoculation of $0.88 L_{50}$ of $R$. typhi shows focal residual rickettsial infection and interstitial pneumonia characterized by lymphohistiocytic infiltration of the alveolar septa; original magnification, $\times 210$. D, Lung of an IFN- $\gamma$ depleted mouse inoculated with the same dose $\left(0.88 \mathrm{LD}_{50}\right)$ of $R$. typhi 5 days earlier shows massive rickettsial infection throughout the microcirculation; original magnification, $\times 53$.

A

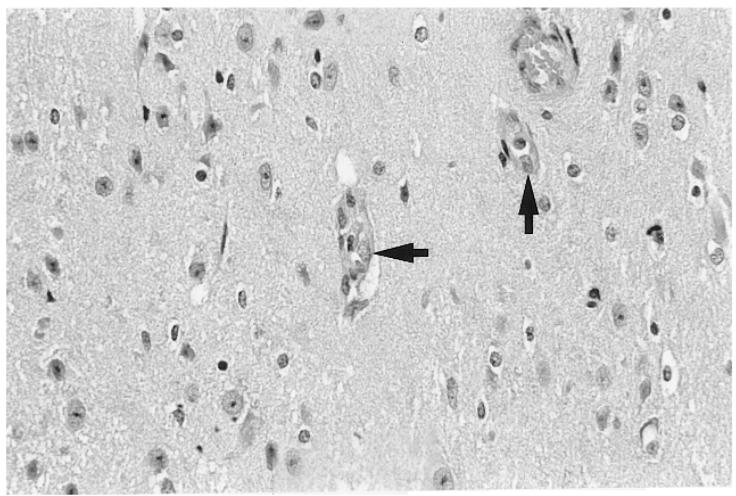

B

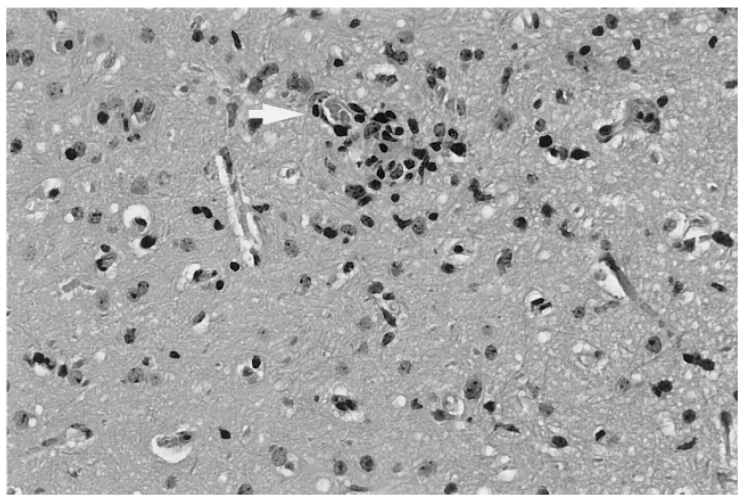

\section{Figure 2.}

A, Photomicrograph of brain of a mouse inoculated with $10 \mathrm{LD}_{50}$ of $R$. typhi 6 days earlier showing the typical acute effect of severe typhus group rickettsial infection, marked cytoplasmic swelling of cerebral endothelial cells (arrowheads); hematoxylin-eosin stain,; original magnification, $\times 210$. B, Photomicrograph of brain of a sham-depleted mouse inoculated 15 days earlier with $0.1 \mathrm{LD}_{50}$ of $R$. typhi demonstrates a classic rickettsia-associated lesion, a typhus nodule consisting of a cerebral blood vessel (white arrowhead) with perivascular infiltration of the neuropil by macrophages and lymphocytes; hematoxylin-eosin stain; original magnification, $\times 105$.

and brain of the sham-depleted mice at titers that were not significantly different $(p>0.05)$ from those assayed on Day 5 (Table 3). The trend toward greater rickettsial content approached statistical significance on Day 5 only for the lungs of mice depleted of IFN- $\gamma$ $(p=0.054)$ or CD8 T lymphocytes $(p=0.074)$ as 




\section{Figure 3.}

Electron photomicrograph of the lung of a mouse infected 2 days earlier with $10 \mathrm{LD}_{50}$ of $R$. typhi. An alveolar capillary endothelial cell contains rickettsiae, some with electron-dense protoplasm (arrowheads) and others with relatively electron-lucent protoplasm (arrows). Bar, $1 \mu \mathrm{m}$.

compared with sham-depleted mice. On Day 10, the rickettsial content of the CD8 T lymphocyte depleted mice was greater than in the sham-depleted mice in the lungs $(p=0.040)$, and approached statistical significance in the spleen $(p=0.08)$. By Day 15 after inoculation, the four killed mice of the sham-depleted mice had cleared their organs of infectious rickettsiae detectable by plaque assay, and the other eight had recovered clinically with return to normal physical appearance and activity. The clearance of organisms in sham-depleted mice on Day 15 compared with detection of low levels of rickettsiae in some of the untreated mice at this time suggests that normal ascites fluid might have conferred some minor protective factor in the sham-depleted mice.

Histopathologic lesions were present during the incubation period on Day 5 before the onset of illness in all groups of animals. Within the liver were multiple foci of inflammation that were randomly distributed in the hepatic lobule and contained a variable mixture of macrophages, neutrophils, and lymphocytes, and frequently one or more hepatocytes with a shrunken, eosinophilic appearance suggestive of apoptosis.

In the sham-depleted mice, $R$. typhi were observed on Day 5 in many alveolar septal capillaries, in focal cerebral and renal intertubular capillary endothelial cells, in numerous splenic macrophages especially within the marginal zones of the periarteriolar lymphoid sheaths, and in the multifocal hepatic inflammatory lesions, as well as in many Kupffer cells. The multifocal vascular infection was not associated with any cellular inflammatory or immune response on Day 5. On Day 10 in the sham-depleted animals, typhus group rickettsiae were observed in the same locations as on Day 5 (Fig. 1C) as well as focally in myocardial capillaries, renal glomeruli, and meningeal blood vessels. Most of the rickettsiae had been cleared from the sham-depleted mice by Day 15 with only focal typhus group rickettsial antigen detected in thickened alveolar septa, in cerebral capillaries in association with perivascular lymphohistiocytic infiltrates, and in Kupffer cells and splenic macrophages.

Many of the most classic lesions of typhus group infection, the so-called glial nodules, which are perivascular infiltrates of the neuropil by macrophages and lymphocytes, were observed in the shamdepleted mice (Fig. 2B). Rickettsial antigen had been cleared from all but rare examples of this lesion on Day 15.

The sham-depleted mice developed a brisk humoral immune response with rise in immunofluorescent assay antibody titer to $R$. typhi from 1:40 on Day 5 to 1:2560-1:5120 on Days 10 and 15. The serum on Day 10 contained $129 \mathrm{pg} / \mathrm{ml}$ of IL-10, but none was detectable on Days 5 and 15 (Fig. 8A). The serum concentration of IL-12 was elevated on Day $5(p=$ $0.015)$, but not on Day 10 ( $p=0.08$ ) or Day 15 (Fig. $8 \mathrm{~B}$ ). IL-2, IL-4, and IFN- $\gamma$ were not detected in serum throughout the experiment. The cytokine levels in the supernatant fluid of spleen cells stimulated with con- 


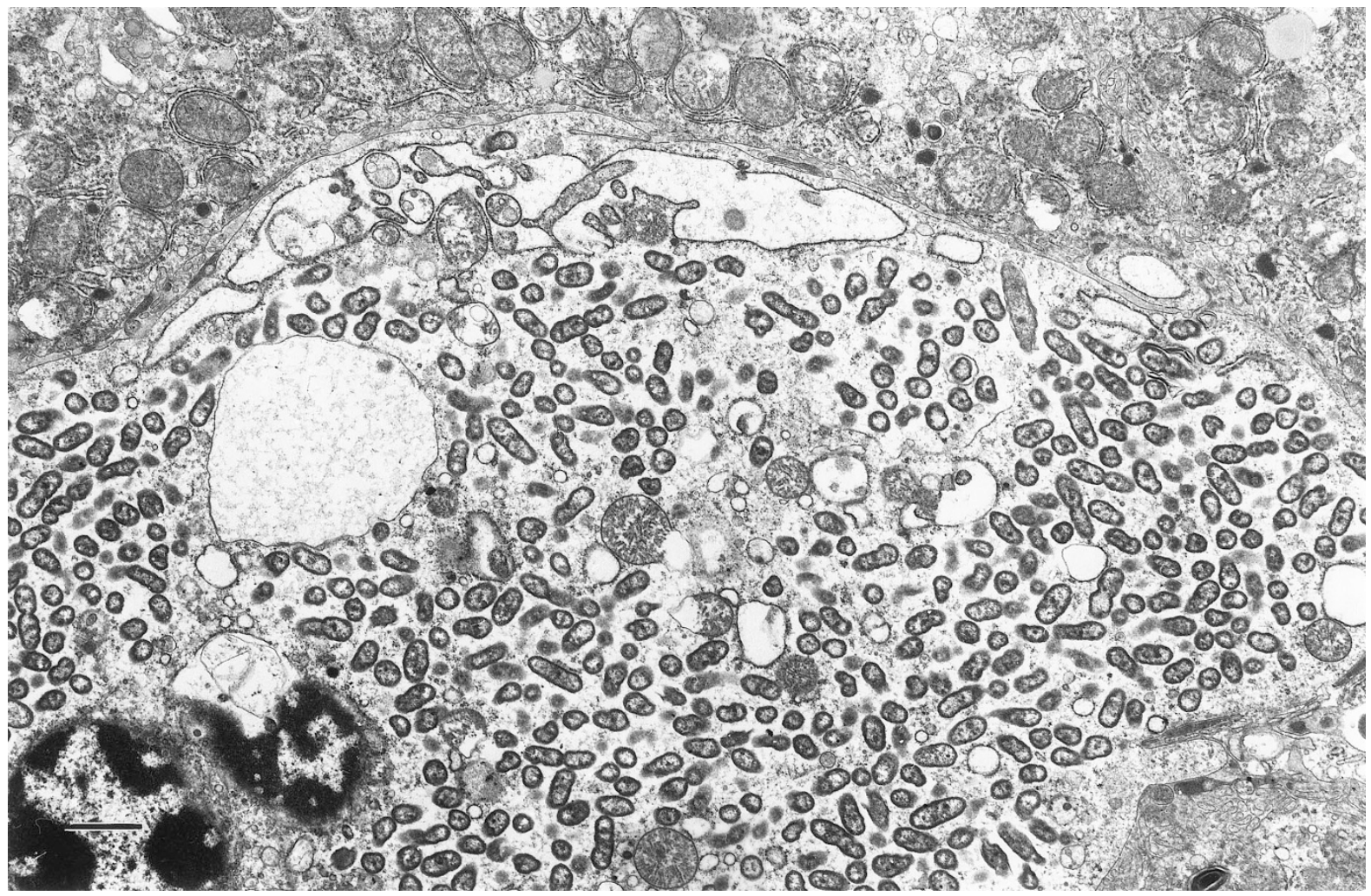

\section{Figure 4}

Electron photomicrograph of mouse liver 4 days after infection with $10 \mathrm{LD}_{50}$ of $R$. typhi shows a Kupffer cell with cytoplasm containing a massive quantity of rickettsiae, swollen mitochondria, and dilated cisterns of rough endoplasmic reticulum. Bar $1 \mu \mathrm{m}$.

canavalin A indicated that $R$. typhi infection caused transient immunosuppression with decreased concentrations of IL-2 and IL-12 in concanavalin-Astimulated splenocyte cultures from infected mice on Days 10 and 15 (Fig. 9).

\section{Comparison of the Mortality, Rickettsial Content in the Organs, and Immunohistochemical Evaluation of the Distribution of R. typhi in Mice Depleted of IFN- $\gamma$ or CD8 $T$ Lymphocytes and in Sham-Depleted Mice}

Mice depleted of IFN- $\gamma$ became ill one day earlier, and $100 \%$ died when infected with a rickettsial dose that killed only $43 \%$ of sham-depleted mice $(p=0.002)$ (Table 2). Death occurred earlier (Day 10) than in sham-depleted mice (median, Day 12.5). Mice depleted of CD8 T lymphocytes also had a significantly higher mortality rate (93\%) than sham-depleted mice $(p=0.011)$. The course of illness resulting in death was shorter than in the sham-depleted mice, similar to that of the IFN- $\gamma$-depleted group.

In the CD8 T lymphocyte-depleted mice, the quantities of $R$. typhi in the organs were impressively increased compared with the sham-depleted animals, as observed by immunohistochemistry. The differences were most dramatic on Day 10 when severe rickettsial infection was observed in pulmonary alveolar capillaries, cerebral capillaries, renal interstitial capillaries, glomeruli, arteries and veins, myocardial capillaries, endocardium, hepatic inflammatory le- sions, and Kupffer cells and in splenic macrophages and blood vessels.

In the IFN- $\gamma$-depleted mice, there were more typhus group rickettsiae observed in the organs by immunohistochemistry on Day 5 than in the other groups of animals. Severe rickettsial infection was present in cerebral, pulmonary alveolar, renal interstitial, and myocardial capillaries and in splenic and hepatic macrophages (Fig. 1D).

The rickettsial organ infectivity titers supported these observations (Table 3), although technical factors intrinsic to the plaque assay for $R$. typhi yielded results with standard deviations too large to achieve statistical significance. None of the IFN- $\gamma$-depleted mice survived until they were scheduled to be killed on Day 10 for analysis of rickettsial infectivity content, antibody, and cytokine responses. The sera of CD8 T lymphocyte-depleted mice surviving on Day 10 had immunofluorescent assay titers to $R$. typhi of 1:1280$1: 2560$.

\section{Discussion}

Investigations of immune mechanisms and of attenuation of typhus group rickettsiae have been impeded by the lack of an animal model in which endothelial cells are identified as the major target, there are histopathologic lesions characteristic of the typhus fevers, and lethal or non-lethal outcome can be reliably predicted by the dose of inoculation. Two useful 


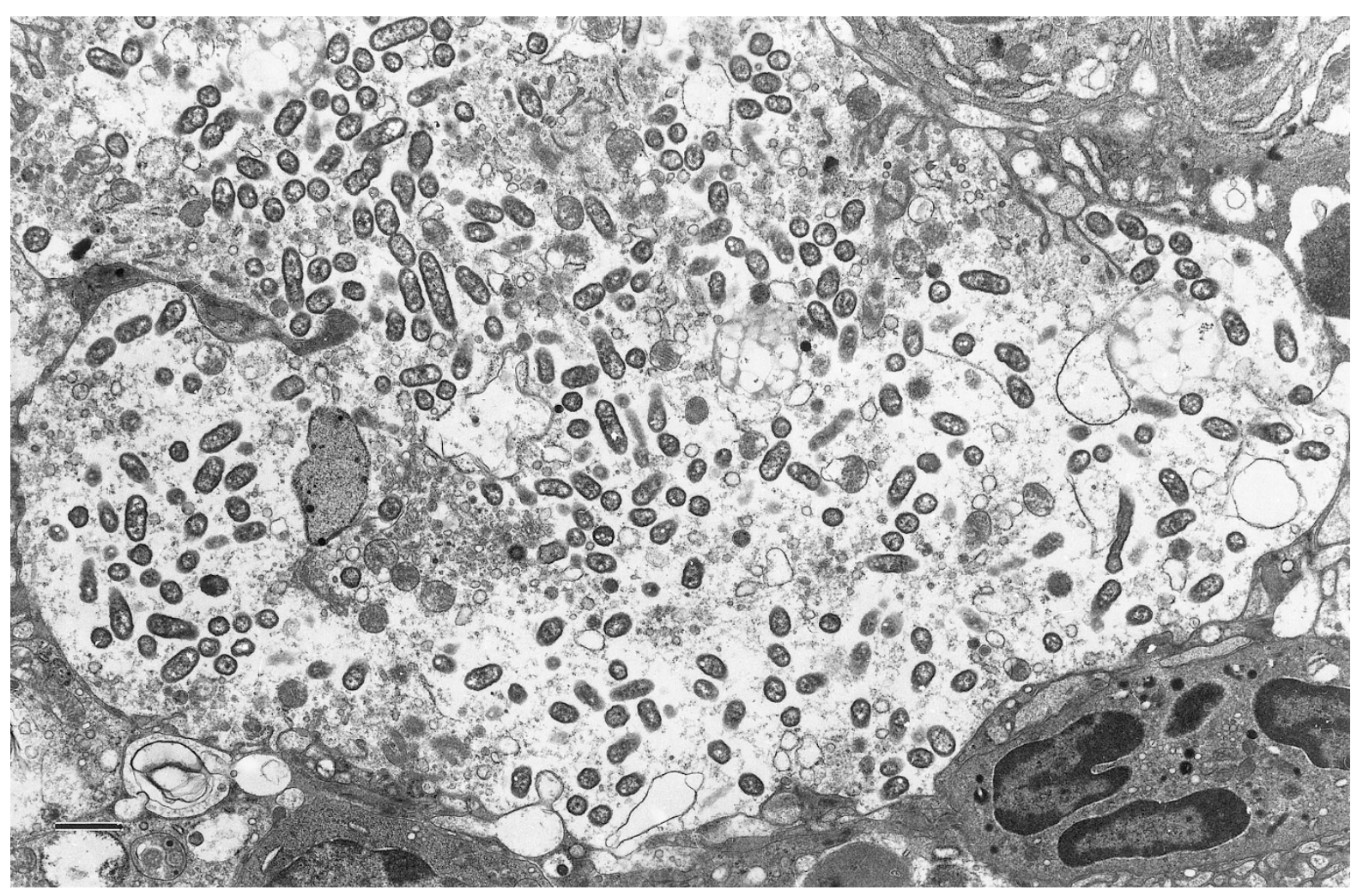

Figure 5.

Electron photomicrograph of spleen of a mouse infected 4 days earlier with $10 L_{50}$ of $R$. typhi shows a heavily infected macrophage. Bar $1 \mu \mathrm{m}$.

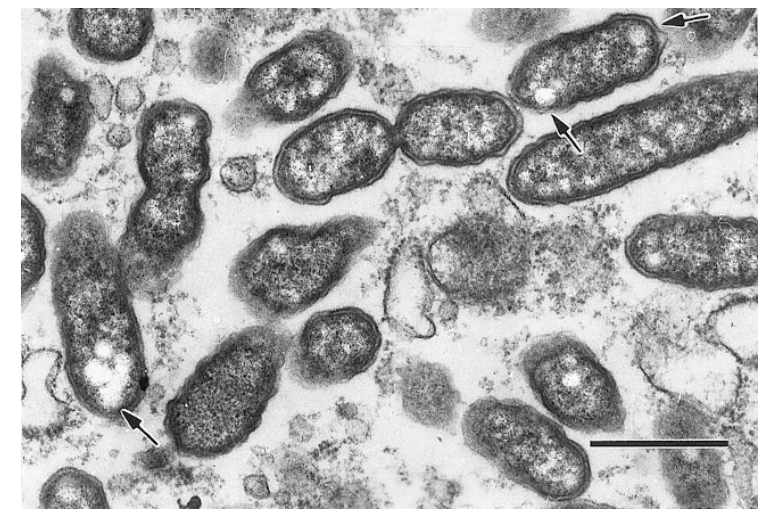

Figure 6.

Electron photomicrograph of intracellular R. typhi containing cytoplasmic vacuoles (arrows). Bar $0.5 \mu \mathrm{m}$.

inbred mouse models of SFG rickettsioses have been developed: Balb/c mice infected intravenously with $R$. australis and $\mathrm{C} 3 \mathrm{H} / \mathrm{HeN}$ mice infected intravenously with R. conorii (Malish 7 strain) (Feng et al, 1993; Walker et al, 1994). The latter model has been utilized to determine the critical importance of IFN- $\gamma$ and CD8 $T$ lymphocytes in immunity to SFG rickettsiae in vivo (Feng et al, 1994, 1997). The lesser ability of typhus group rickettsiae to spread from cell-to-cell and the greater propensity to establish prolonged or latent infection in nature (eg, R. prowazekii in humans and flying squirrels and $R$. typhi in rats) suggests that typhus group rickettsiae may interact differently with

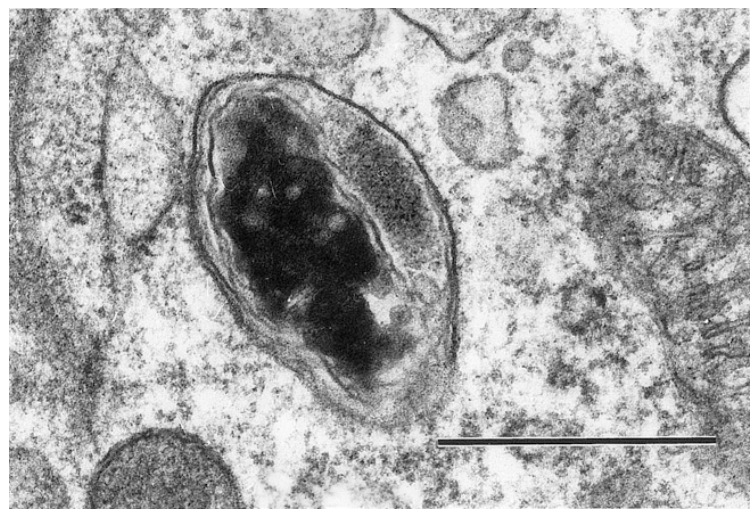

Figure 7.

Electron photomicrograph of a necrotic-appearing rickettsia with dense homogeneous protoplasm and an irregular cell wall within a phagolysosome of a splenic macrophage 4 days after inoculation of $10 \mathrm{LD}_{50}$ of $R$. typhi. Bar 0.5 $\mu \mathrm{m}$.

the immune system (Bozeman et al, 1975; Wisseman and Waddell, 1975; Sonenshine et al, 1978; Traub et al, 1978). In this study, the critical importance of IFN- $\gamma$ and CD8 T lymphocytes has been linked to survival of the host and clearance of typhus group rickettsiae, similar to what has been documented for the SFG rickettsia, $R$. conorii. This advance in knowledge was enabled by the development and characterization of a mouse model for typhus group rickettsial infection.

The importance of cellular immunity to $R$. typhi has been demonstrated previously in two animal models. 
Table 2. Morbidity and Mortality of C3H/HeN Mice Infected with $\boldsymbol{R}$. Typhi and Depleted of Gamma Interferon or CD8 T Lymphocytes

\begin{tabular}{|c|c|c|}
\hline Experimental treatment & Day of onset & $\begin{array}{c}\text { Mortality }{ }^{a} \\
\text { (Day of death) }\end{array}$ \\
\hline \multicolumn{3}{|l|}{ Experiment 1} \\
\hline Sham-depleted $\left(n=10,6\right.$ killed on schedule $\left.{ }^{b}\right)$ & D 9 & $2 / 4(d .13)$ \\
\hline IFN- $\gamma$ depleted ( $\mathrm{n}=10,2$ killed on schedule) & D 8 & $8 / 8(\mathrm{~d} .10)$ \\
\hline CD8-depleted ( $n=10,4$ killed on schedule) & D 9 & $5 / 6(d .12)$ \\
\hline \multicolumn{3}{|l|}{ Experiment 2} \\
\hline Sham-depleted $\left(n=10,4\right.$ killed on schedule $\left.{ }^{a}\right)$ & D 8 & $2 / 6(d .10)$ \\
\hline IFN- $\gamma$ depleted ( $n=6,2$ killed on schedule) & D 7 & $4 / 4(d .10)$ \\
\hline CD8-depleted ( $n=8,3$ killed on schedule) & D 8 & $5 / 5(d .10)$ \\
\hline \multicolumn{3}{|l|}{ Experiment 3} \\
\hline Sham-depleted $\left(n=10,6\right.$ killed on schedule $\left.{ }^{a}\right)$ & D 9 & 2/4 (d.12,d.13) \\
\hline IFN- $\gamma$ depleted ( $n=6,2$ killed on schedule) & D 8 & $4 / 4(d .10)$ \\
\hline CD8-depleted ( $n=8,4$ killed on schedule) & D 9 & $4 / 4(d .10)$ \\
\hline \multicolumn{3}{|l|}{, } \\
\hline Sham-depleted & median, d. 9 & $43 \%$ (median, $12.5 \mathrm{~d}$.) \\
\hline IFN- $\gamma$ depleted & median, d. 8 & $100 \%^{c}$ (d. 10$)$ \\
\hline CD8-depleted & median, d.9 & $93 \%^{d}$ (median, d. 10$)$ \\
\hline
\end{tabular}

${ }^{a}$-Number of animals that died of rickettsial infection/number of animals infected with $R$. typhi.

${ }^{b}$-Two animals in each group were scheduled for killing and collection of blood and organs on Days 5, 10, and 15. Some IFN- $\gamma$ and CD8-depleted animals died before scheduled time for killing.

${ }^{c}$-Statistically significantly $(p=0.002)$ higher mortality than sham-depleted mice.

${ }^{d}$-Statistically significantly $(p=0.011)$ higher mortality than sham-depleted mice.

Table 3. Infectious Content ${ }^{a}$ of Rickettsia Typhi in Mice with Depletion of IFN- $\gamma$ or CD8 ${ }^{+}$T-Cells

\begin{tabular}{ccccc}
\hline Day after infection & Group & Spleen & Lungs & Brain \\
\hline 5 & sham-depleted & $39 \pm 16.9$ & $131.9 \pm 71.8$ & $1.1 \pm 1.5$ \\
& IFN- $\gamma$ depleted & $59.8 \pm 20^{b}$ & $393.3 \pm 147.9^{c}$ & $6.7 \pm 6.1^{b}$ \\
& CD8 $^{+}$depleted & $67.8 \pm 19.4^{b}$ & $357.1 \pm 145.3^{d}$ & UD $^{e}$ \\
10 & sham-depleted & $134.2 \pm 118.6$ & $131.9 \pm 9.8$ & $4.0 \pm 5.7$ \\
& IFN- $\gamma$ depleted & $\mathrm{ND}^{f}$ & $\mathrm{ND}$ & ND \\
& CD8 $^{+}$depleted & $304.2 \pm 54.2^{g}$ & $309.8 \pm 101.1^{h}$ & UD \\
15 & sham-depleted & UD & UD & UD \\
& IFN- $\gamma$ depleted & ND & ND & ND \\
& CD8 $8^{+}$depleted & UD & UD & UD \\
\hline
\end{tabular}

${ }^{a}-\mathrm{PFU} \times 10^{2} / \mathrm{gm}$ tissue.

${ }^{b}$-The quantity of rickettsiae was not significantly different than in the same organ in the sham-depleted group at this time.

${ }^{c}$-Compared with lungs of sham-depleted mice, $p=0.054$.

${ }^{d}$ Compared with lungs of sham-depleted mice, $p=0.074$.

$e-U D$, undetectable.

${ }^{f}-\mathrm{ND}$, not determined, all mice died of infection before this time point.

$g$-Compared with spleen of sham-depleted mice, $p=0.08$.

${ }^{h}$-Compared with lungs of sham-depleted mice, $(p=0.040)$.

Murphy et al inoculated guinea pigs intradermally with $8.2 \times 10^{4}$ or $8.2 \times 10^{6}$ plaque-forming units (PFU) of $R$. typhi and observed the development of an eschar containing predominantly large mononuclear cells with peak rickettsial content on Day 3 and markedly reduced but persistent infection through the final observation on Day 7 (Murphy et al, 1978). Immune animals cleared the inoculated rickettsiae rapidly in association with infiltration of large mononuclear cells and lymphocytes and did not develop eschars. Adoptive transfer of immune spleen cells from inbred guinea pigs into naive syngeneic strain 13 guinea pigs resulted in inhibition of the growth of intradermally inoculated R. typhi (Murphy et al, 1979). In contrast, passive transfer of immune serum did not prevent the growth of the inoculated rickettsiae. In this guinea pig model, rickettsiae were observed by immunofluorescence to be associated with the microvasculature.

Crist et al investigated rickettsial immunity in Balb/c mice inoculated subcutaneously with $R$. typhi. Control of infection at the inoculation site preceded reduction of the infectious rickettsial content in the spleen where viable organisms persisted for at least 28 days (Crist et al, 1984a). Adoptive transfer of immune spleen cells, but not passive transfer of immune sera, resulted in earlier control of the growth of $R$. typhi in the spleen of subcutaneously infected animals. Depletion of lymphoid cells with antibodies to theta antigen suggested 
A

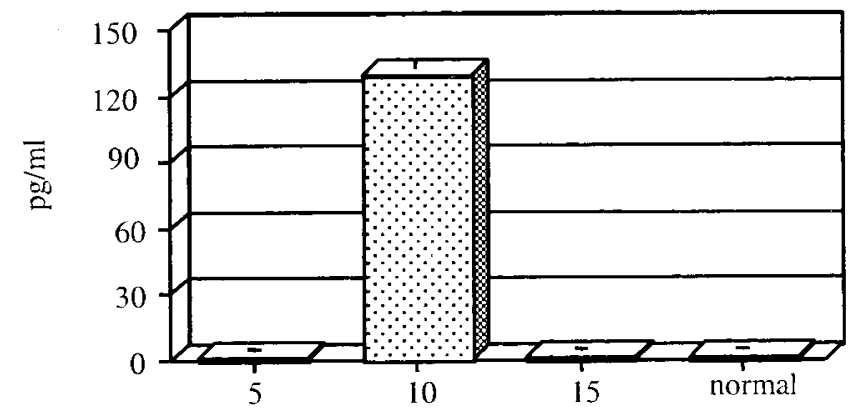

Day after inoculation

B

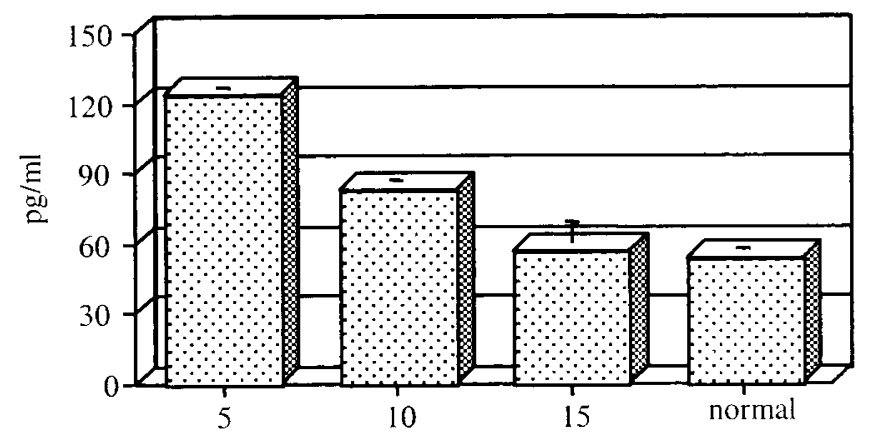

Day after inoculation

Figure 8.

Serum cytokine levels of sham-depleted $\mathrm{C} 3 \mathrm{H} / \mathrm{HeN}$ mice infected with $0.88 \mathrm{LD}_{50}$ of $R$. typhi on Days 5,10 , and 15 and in normal mice. A, Concentration of serum IL-10. B, Concentration of serum IL-12.

the importance of T lymphocytes in immunity to $R$. typhi (Crist et al, 1984b).

The other contemporary studies of immunity that have defined the importance of IFN- $\gamma$ in rickettsial killing and the existence of a subpopulation of T lymphocytes that is a potentially important mediator of immunity to typhus group rickettsial infections have been performed exclusively in vitro. Investigations demonstrating that IFN- $\gamma$ and TNF- $\alpha$ activate the intracellular killing of $R$. prowazekii by nitric oxide-dependent and nitric oxideindependent mechanisms have been conducted principally in fibroblasts and macrophages, which are non-targets and minor targets, respectively, in $R$. prowazekii and $R$. typhi infections rather than in the principal target of infection, the endothelium (Turco and Winkler, 1983a, 1983b, 1988, 1993; Wisseman and Waddell, 1983). The importance of IFN- $\gamma$ in immunity to typhus group rickettsiae had not been evaluated in vivo before the present study. Similarly, this is the first report that demonstrates the importance of a particular T lymphocyte subset in immunity to typhus group rickettsiae. The demonstration of the critical importance of CD8 T lymphocytes suggests that previously described major histocompatibility class I-restricted cytotoxic T lymphocytes in $R$. typhiimmune mice might be important effectors in the clearance of rickettsiae and recovery from infection (Rollwagen et al, 1986).

The evaluation in vivo of the role of phenomena that are described in vitro is an essential step in the elucidation of mechanisms of immunity and pathogenesis. Unfortunately, ideal or even near ideal models are not available for many infectious diseases. The $\mathrm{C} 3 \mathrm{H} /$ HeN mouse- $R$. typhi model represents a useful addition to the portfolio of tools to study immunity and pathogenesis in typhus group rickettsial infection. It accurately reproduces most of the important features of human typhus fevers, namely hematogenous dissemination; endothelial cell infection as the principal target; macrophage infection as a minor target; characteristic vascular pathology (endothelial swelling, perivascular infiltration of foci of infection by lymphocytes and macrophages); typical organ involvement including the most clinically relevant manifestations, rickettsial encephalitis and pneumonitis; predictable lethal and sublethal doses; and clearance of rickettsiae associated with perivascular lymphohistiocytic infiltrate (Wolbach et al, 1922; Walker et al, 1989). The mouse host offers the availability of numerous immunologic reagents and gene knockout animals for further investigations. The two major reservations about this model are the bypass of the cutaneous portal of 
A

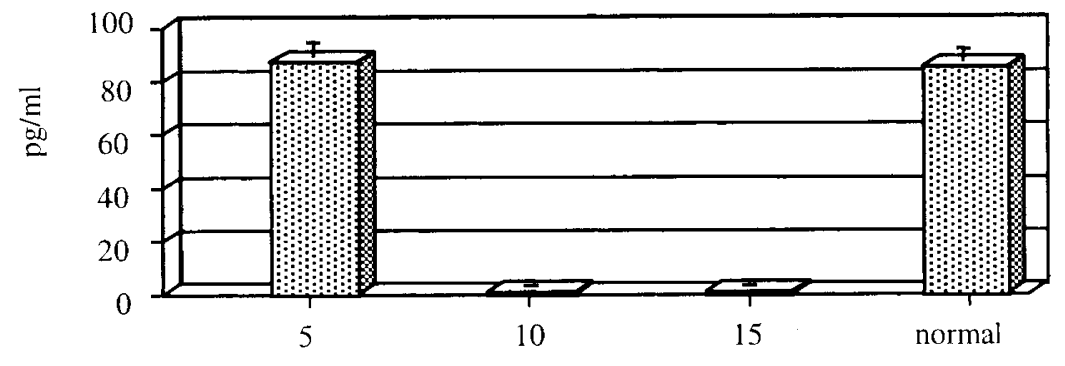

Day after inoculation

B

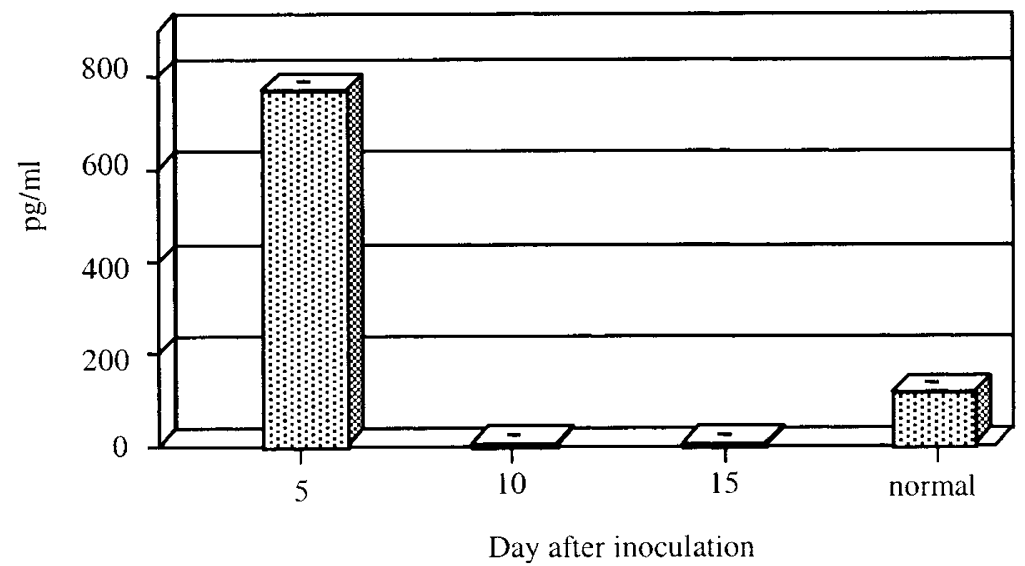

Figure 9.

Cytokine levels in the supernatant fluid of concanavalin-A-stimulated spleen cells from normal C3H/HeN mice and mice infected with $0.88 \mathrm{LD}_{50}$ of $R$. typhi 5 , 10, and 15 days earlier. A, Concentration of IL-2. B, Concentration of IL-12.

entry (associated in naturally acquired infections with skin contamination by infected insect feces) and the host species differences between humans and mice.

Contemporary considerations include the very grave threat that typhus group rickettsiae would pose if used as bioterrorist agents, particularly if resistance to tetracycline and chloramphenicol were engineered into the rickettsiae, as has been done with rifampin (Rachek et al, 1998). Indeed, $R$. prowazekii and $R$. rickettsii are among the 12 bacterial species on the list of agents with restrictions on transfer because of legislation to prevent bioterrorism (Atlas, 1998), and $R$. prowazekii has been produced and stockpiled as a biologic weapon (Alibek and Handelman, 1999). In a review article on $R$. prowazekii, two of the leading investigators of immunity to typhus rickettsiae, Winkler and Turco, stated, "Lack of an animal model (other than the cotton rat and the cynomolgus monkey) which reproduces the features of human epidemic typhus has limited the study of host defenses against this organism" (Winkler and Turco, 1988). The present model, although utilizing $R$. typhi rather than $R$. prowazekii, would enable investigation of the role of selected genes in attenuation. The entire genome of $R$. prowazekii has been sequenced and is available, and there is a very close evolutionary and genetic relationship between $R$. prowazekii and $R$. typhi (Roux and Raoult, 1995; Stothard and Fuerst, 1995; Roux et al, 1997; Anderson et al, 1998). Gene sequences from $R$. prowazekii could be used to identify the corresponding gene in $R$. typhi. Deletion of the gene by homologous recombination is within the reach of contemporary technologic approaches to rickettsiae, as indicated by the successful transformation of $R$. typhi by electroporation (Troyer et al, 1999). $R$. typhi with gene deletion could be tested in the $\mathrm{C} 3 \mathrm{H} / \mathrm{HeN}-R$. typhi model for attenuation and for the ability to stimulate immunity. Thus a more powerful approach is now available, not only for the study of immunity, but also for vaccine development and the investigation of pathogenic mechanisms of these rickettsiae.

\section{Materials and Methods}

\section{Rickettsia}

R. typhi (Wilmington strain) was obtained from the American Type Culture Collection (Rockville, MD). Embryonated chicken eggs were inoculated, and the infectious yolk sacs were harvested and pooled. The 
$10 \%$ yolk sac stock contained $10^{6.2}$ LD $_{50}$ per $\mathrm{ml}$. Our titrations demonstrated that approximately $3 \times 10^{4}$ PFU of $R$. typhi was $1 L_{50}$, and a previous study demonstrated that $1 \mathrm{PFU}$ was equivalent to 1 median tissue culture infectious dose $\left(\operatorname{TCID}_{50}\right)$ (Ormsbee et al, 1978).

\section{Cytokine Assays}

ELISA kits (Genzyme, Cambridge, MA) were used to measure the concentrations of IL-2, IL-4, IL-10, IL-12, and IFN- $\gamma$. The sera used for studies of cytokine levels were collected on Days 5, 10, and 15 from different groups of infected mice. Splenocytes were harvested on Days 5, 10, and 15 after rickettsial inoculation, and $5 \times 10^{6}$ cells $/ \mathrm{ml}$ were stimulated with concanavalin A (5 $\mu \mathrm{g} / \mathrm{ml}$ ). After incubation at $37^{\circ} \mathrm{C}$ in a $5 \% \mathrm{CO}_{2}$ atmosphere for 48 hours, the culture supernatants were harvested and centrifuged at $440 \times g$ for 10 minutes. The supernatants were stored at $-20^{\circ} \mathrm{C}$ until assayed for cytokine levels. The cytokine levels in the sera and supernatants were assayed by ELISA, performed according to the manufacturer's instructions.

\section{Quantification of the Infectious Content of R. typhi in Tissues by Plaque Assay and Cell Culture Titration}

The plaque assay method was similar to that described previously for $R$. conorii (Walker et al, 1994). Because plaque formation by $R$. typhi was slower, the results were read on Day 8 instead of on Day 3 as is the case for $R$. conorii. To prevent drying of the agarose overlay, $1 \mathrm{ml}$ of Eagle's minimum essential medium (EMEM; Gibco, Gaithersburg, MD) containing $1 \%$ fetal bovine serum (FBS; Hyclone, Logan, UT) and $1 \mu \mathrm{g} / \mathrm{ml}$ cycloheximide (Sigma, St. Louis, MO) was added after the agarose solidified.

The $\mathrm{TCID}_{50}$ of each sample was determined by inoculation of $0.2 \mathrm{ml}$ of serial 10-fold dilutions of triturated weighed organ samples in duplicate to monolayers of Vero cells in 24-well plates. After incubation at $37^{\circ} \mathrm{C}$ for 2 hours in a $5 \% \mathrm{CO}_{2}$ atmosphere to allow rickettsial attachment and entry, $1 \mathrm{ml}$ of MEM containing $1 \%$ bovine calf serum was added to each well, and the cultures were incubated as above for 10 days. Then the cells were scraped from each well, centrifuged by cytospin (Shandon, Pittsburgh, PA), and stained with Diff-Quik to detect growth of $R$. typhi. $\mathrm{TCID}_{50}$ was calculated for each specimen by the Reed-Muench equation.

\section{Experimental Design}

In the first set of experiments to determine the rickettsial and pathologic events in the lethal and sublethal infections, 44 six-to-eight-week-old male $\mathrm{C} 3 \mathrm{H} / \mathrm{HeN}$ mice were divided into two groups: 20 mice were inoculated intravenously with $10 \mathrm{LD}_{50}$ of $R$. typhi, and 24 mice were inoculated intravenously with $0.1 L_{50}$. Of the mice inoculated with 10 LD $_{50}$, four were killed on Day 2, six on Day 4, and six on Day 6, and four were observed for mortality. Of the mice inoculated with 0.1 $\mathrm{LD}_{50}$, six were killed on each of Days 5,10 , and 15, and the remaining six were observed for 4 weeks for mortality. Sera were collected for antibody titration, and spleen, lung, and brain were collected aseptically for titration of infectious rickettsial content by determining the $\mathrm{TCID}_{50}$.

In the second set of experiments to evaluate the roles of IFN- $\gamma$ and CD8 T lymphocytes in immunity, a total of 78 six-to-eight-week-old male $\mathrm{C} 3 \mathrm{H} / \mathrm{HeN}$ mice were used in three replicated experiments: 30 mice for experiment 1, and 24 mice for each of replicates 2 and 3. In each replicate, the mice were divided into three groups, and inoculated intravenously with $0.88 L_{50}$ of $R$. typhi via the tail vein. In group 1, 30 mice (10 in each of replicates 1,2 , and 3 ) received ascites induced by the injection of the murine non-immunoglobulin secreting myeloma SP2/0 cells as the sham-depleted group. In group 2 (IFN- $\gamma$ depleted), 22 of the mice (10 in experiment 1 , and six in each of replicates 2 and 3 ) received ascites induced by hybridoma R4-6A2 (HB170, ATCC) at $1 \mathrm{mg}$ of protein per mouse per dose on Day 0 and Day 2 after $R$. typhi inoculation. In group 3 (26 mice; 10 for experiment 1, and eight for each of replicates 2 and 3 ), $\mathrm{CD}^{+} \mathrm{T}$ lymphocytes were depleted by the inoculation of ascites induced by hybridoma 53-6.72 (TIB-105, ATCC) at $1 \mathrm{mg}$ of protein per mouse per dose on the day of rickettsial infection and 3 days before inoculation. The globulin fraction was purified from ascites fluids by ammonium sulfate precipitation.

All mice were observed daily to determine the morbidity and mortality. On each of Days 5, 10, and 15 after inoculation with $R$. typhi, two surviving mice, if any, in each group were killed, sera were collected and stored at $-20^{\circ} \mathrm{C}$ for measurement of antibody titers by indirect immunofluorescent assay and for cytokine assays. Samples of spleen, lungs, and brain were weighed and used for rickettsial infectious titration by plaque assay, and part of the spleen was used for concanavalin A stimulation of lymphocytes and measurement of cytokine secretion. Samples of spleen, lungs, heart, liver, kidney, brain, testis, and testicular adnexae were fixed in $4 \%$ neutral buffered formaldehyde for histopathology and immunohistochemical observation of the anatomic distribution and relative quantities of $R$. typhi.

\section{Statistical Analysis}

All statistical evaluations were performed using Student's $t$ test.

\section{Histopathology and Immunohistochemistry}

Tissue sections were prepared and stained by hematoxylin-eosin and by immunohistochemistry with a monoclonal antibody for typhus group rickettsial lipopolysaccharide, as previously described (Walker et al, 1997).

\section{Electron Microscopy}

Pieces of tissues were placed immediately into a drop of fixative and cut into $1-\mathrm{mm}^{3}$ cubes. They were then 
fixed in a mixture of $1.25 \%$ formaldehyde, $2.5 \%$ glutaraldehyde, and $0.035 \mathrm{~m}$ trinitrophenol with $0.03 \%$ $\mathrm{CaCl}_{2}$ in $0.05 \mathrm{M}$ cacodylate buffer $\mathrm{pH} 7.3$ (Ito and Rikihisa, 1981), postfixed in $1 \% \mathrm{OsO}_{4}$ in the same buffer, stained en bloc with 1\% uranyl acetate in $0.1 \mathrm{M}$ maleate buffer, $\mathrm{pH} 5.2$, processed through a graded series of concentrations of ethanol, and embedded into Poly/Bed 812. Ultrathin sections were cut on a Sorvall MT-6000 ultramicrotome, stained with uranyl acetate and lead citrate, and examined in a Philips 201 electron microscope at an accelerating voltage of 60 $\mathrm{kV}$.

\section{Acknowledgements}

The authors wish to thank Tom Bednarek for assistance in the preparation of the figures, Violet Han for her assistance in the preparation of electron micrographs, Gui-min He for statistical analysis, and Josie Ramirez-Kim for expert secretarial assistance in the preparation of this manuscript. This research was supported by a grant from the National Institute of Allergy and Infectious Diseases (Al 21242).

\section{References}

Anderson SGE, Zomorodipour A, Andersson JO, SicheritzPonten T, Alsmark UCM, Podowski RM, Naslund AK, Eriksson A-S, Winkler HH, and Kurland CG (1998). The genome sequence of Rickettsia prowazekii and the origin of mitochondria. Nature 396:133-140.

Alibek K and Handelman S (1999). Biohazard: The chilling true story of the largest covert biological weapons program in the world, told from the inside by the man who ran it. New York: Random House, 70-86.

Atlas RM (1998). Biological weapons pose challenge for microbiology community. ASM News 64:383-389.

Bozeman FM, Masiello SA, Williams MS, and Elisberg BL (1975). Epidemic typhus rickettsiae isolated from flying squirrels. Nature 255:545-547.

Crist AE Jr, Wisseman CL Jr, and Murphy JR. (1984a). Characteristics of Rickettsia mooseri infection of normal and immune mice. Infect Immun 43:38-42.

Crist AE Jr, Wisseman CL Jr, and Murphy JR. (1984b). Characteristics of lymphoid cells that adoptively transfer immunity to Rickettsia mooseri infection in mice. Infect Immun 44:55-60.

Feng H-M, Popov VL, and Walker DH (1994). Depletion of gamma interferon and tumor necrosis factor alpha in mice with Rickettsia conorii-infected endothelium: Impairment of rickettsicidal nitric oxide production resulting in fatal, overwhelming rickettsial disease. Infect Immun 62:1952-1960.

Feng H-M, Popov VL, Yuoh G, and Walker DH (1997). Role of $T$ lymphocyte subsets in immunity to spotted fever group rickettsiae. J Immunol 158:5314-5320.

Feng H-M, Wen J, and Walker DH (1993). Rickettsia australis infection: A murine model of a highly invasive vasculopathic rickettsiosis. Am J Pathol 142:1471-1482.

Heinzen RA, Hayes SF, Peacock MG, and Hackstadt T (1993). Directional actin polymerization associated with spot- ted fever group rickettsia infection of vero cells. Infect Immun 61:1926-1935.

Ito S and Rikihisa T (1981). Techniques for electron microscopy of rickettsiae. In: Burgdorfer W and Anacker RL, editors. Rickettsiae and rickettsial diseases. New York: Academic Press, 213-227.

Murphy JR, Wisseman CL Jr, and Fiset P (1978). Mechanisms of immunity in typhus infection: Some characteristics of intradermal Rickettsia mooseri infection of guinea pigs. Infect Immun 21:417-424.

Murphy JR, Wisseman CL Jr, and Fiset P (1979). Mechanisms of immunity in typhus infection: Adoptive transfer of immunity to Rickettsia mooseri. Infect Immun 24:387-393.

Ormsbee R, Peacock M, Gerloff R, Tallent G, and Wike D (1978). Limits of rickettsial infectivity. Infect Immun 19:239245.

Rachek LI, Tucker AM, Winkler HH, and Wood DO (1998). Transformation of Rickettsia prowazekii to rifampin resistance. J Bacteriol 180:2118-2124.

Rollwagen FM, Dasch GA, and Jerrells TR (1986). Mechanisms of immunity to rickettsial infection: Characterization of a cytotoxic effector cell. J Immunol 136:1418-1421.

Roux V and Raoult D (1995). Phylogenetic analysis of the genus Rickettsia by $16 \mathrm{~S}$ rDNA sequencing. Res Microbiol 146:385-396.

Roux V, Rydkina E, Eremeeva M, and Raoult D (1997). Citrate synthase gene comparison, a new tool for phylogenetic analysis, and its application for the rickettsiae. Int $\mathrm{J}$ Syst Bacteriol 47:252-261.

Sonenshine DE, Boseman FM, Williams MS, Masiello SA, Chadwick DP, Stocks NI, Lauer DM, and Elisberg BL (1978). Epizootiology of epidemic typhus (Rickettsia prowazekii) in flying squirrels. Am J Trop Med Hyg 27:339-349.

Stothard DR and Fuerst PA (1995). Evolutionary analysis of the spotted fever and typhus groups of rickettsia using 16S rRNA gene sequences. Syst Appl Microbiol 18:52-61.

Teysseire N, Chiche-Portiche C, and Raoult D (1992). Intracellular movements of Rickettsia conorii and R. typhi based on actin polymerization. Res Microbiol 143:821-829.

Traub R, Wisseman CL, and Farhang-Azad A (1978). The ecology of murine typhus: A critical review. Trop Dis Bull 75:237-317.

Troyer JM, Radulovic S, and Azad AF (1999). Green fluorescent protein as a marker in Rickettsia typhi transformation. Infect Immun 67:3308-3311.

Turco $\mathrm{J}$ and Winkler HH. (1983a). Cloned mouse interferongamma inhibits the growth of Rickettsia prowazekii in cultured mouse fibroblasts. J Exp Med 158:2159-2164.

Turco J and Winkler HH. (1983b). Inhibition of the growth of Rickettsia prowazekii in cultured fibroblasts by lymphokines. J Exp Med 157:974-986.

Turco J and Winkler HH (1988). Interactions between Rickettsia prowazekii and cultured host cells: Alterations induced by gamma interferon. In: Byrne GI and Turco J, editors. Interferon and nonviral pathogens. New York: Marcel Dekker, 95-115.

Turco J and Winkler HH (1993). Role of nitric oxide synthase pathway in inhibition of growth of interferon-sensitive and interferon-resistant Rickettsia prowazekii strains in L929 
cells treated with tumor necrosis factor alpha and gamma interferon. Infect Immun 61:4317-4325.

Vishwanath S (1991). Antigenic relationships among the rickettsiae of the spotted fever and typhus groups. FEMS Microbiol Lett 81:341-344.

Walker DH, Feng H-M, Ladner S, Billings AN, Zaki SR, Wear DJ, and Hightower B (1997). Immunohistochemical diagnosis of typhus rickettsioses using an anti-lipopolysaccharide monoclonal antibody. Mod Pathol 10:1038-1042.

Walker DH, Parks FM, Betz TG, Taylor JP, and Muehlberger JW (1989). Histopathology and immunohistologic demonstration of the distribution of Rickettsia typhi in fatal murine typhus. Am J Clin Pathol 91:720-724.

Walker DH, Popov VL, Wen J, and Feng H-M (1994). Rickettsia conorii infection of $\mathrm{C} 3 \mathrm{H} / \mathrm{HeN}$ mice: A model of endothelial-target rickettsiosis. Lab Invest 70:358-368.

Winkler HH and Turco J (1988). Rickettsia prowazekii and the host cell: Entry, growth, and control of the parasite. Curr Top Microbiol Immunol 138:81-107.
Wisseman CL Jr, Edlinger EA, Waddell AD, and Jones MR (1976). Infection cycle of Rickettsia rickettsii in chicken embryo and L-929 cells in culture. Infect Immun 14:1052-1064.

Wisseman CL Jr and Waddell A (1983). Interferon-like factors from antigen- and mitogen-stimulated human leukocytes with antirickettsial and cytolytic actions on Rickettsia prowazekii. Infected human endothelial cells, fibroblasts, and macrophages. J Exp Med 157:1780-1793.

Wisseman CL Jr and Waddell AD (1975). In vitro studies on rickettsia-host cell interactions: Intracellular growth cycle of virulent and attenuated Rickettsia prowazekii in chicken embryo cells in slide chamber cultures. Infect Immun 11:13911401.

Wolbach SB, Todd JL, and Palfrey FW (1922). The etiology and pathology of typhus. Cambridge: League of Red Cross Societies at the Harvard University Press, 152-193.

Zinsser H (1935). Rats, lice and history. New York: Little, Brown, and Company, 1-301. 